\title{
A Combined Attack-Tree and Kill-Chain Approach to Designing Attack-Detection Strategies for Malicious Insiders in Cloud Computing
}

\author{
Adrian Duncan, Sadie Creese, Michael Goldsmith \\ Department of Computer Science, University of Oxford, UK
}

\{firstname.lastname\}@cs.ox.ac.uk

\begin{abstract}
Attacks on cloud-computing services are becoming more prevalent with recent victims including Tesla, Aviva Insurance and SIM-card manufacturer Gemalto[1]. The risk posed to organisations from malicious insiders is becoming more widely known about and consequently many are now investing in hardware, software and new processes to try to detect these attacks. As for all types of attack vector, there will always be those which are not known about and those which are known about but remain exceptionally difficult to detect particularly in a timely manner. We believe that insider attacks are of particular concern in a cloud-computing environment, and that cloudservice providers should enhance their ability to detect them by means of indirect detection.

We propose a combined attack-tree and kill-chain based method for identifying multiple indirect detection measures. Specifically, the use of attack trees enables us to encapsulate all detection opportunities for insider attacks in cloud-service environments. Overlaying the attack tree on top of a kill chain in turn facilitates indirect detection opportunities higher-up the tree as well as allowing the provider to determine how far an attack has progressed once suspicious activity is detected.

We demonstrate the method through consideration of a specific type of insider attack - that of attempting to capture virtual machines in transit within a cloud cluster via use of a network tap, however, the process discussed here applies equally to all cloud paradigms.
\end{abstract}

Keywords: Insider Attacks, Packet Sniffing, Kill Chain, Undetectable Attacks, Malicious Insider, Attack Trees, Indirect Detection.

\section{INTRODUCTION}

Statistics on cyber attacks are rarely consistent and arguably always a best-guess, as they can only reflect attacks that are reported; many successful attacks are not reported and, still more, must go undetected. The Ponemon Institute's Cost of Data Breach Study published in March 2012 [2] reports that the number of attacks from insiders is approximately $33 \%$ of all recorded attacks. Whereas Verizon's 2017 comparable report [3] shows a lower proportion of $25 \%$ up from 14\% in 2013 [4], which was double the actual number of actual attacks in the previous year. These figures show that the problem of insider attacks has not improved in the last five years and as an industry, we are yet to get the problem under control.

The number of detected attacks is obviously only one metric by which to measure the scale of cyber attack, another is the actual cost of successful attacks. A survey by Ponemon released in November, 2012 [5, p. 3] states that malicious insiders accounted for $78 \%$ of the total cost of all cyber crime reported. Their corresponding 2016 report [6] showed an increase in the number of attacks from 2012 in 2015 and 2016. The Computer Security Institute's annual survey of organisations that have suffered actual breaches suggests that up to $50 \%$ of losses from attacks are caused through insiders based on their figures for 2010 / 2011. The comporable report from 2017 shows that malicious insiders are the most expensive attack category when weighted by attack frequency, however the actual value had fallen slighly over the previous 12 months [7, 8]

Regardless of which statistics you believe, if any, it is clear that insider attacks are a potential source of significant threat and associated risk, warranting resources to be deployed in development of protection mechanisms. We are particularly concerned with the potential for insider attacks in cloud service environments, as we believe there are various economic reasons, among others, to consider cloud as highly attractive to threat sources (in general).

Attacks on cloud-computing services are becoming more prevalent with recent victims including Tesla, Aviva Insurance and SIM-card manufacturer Gemalto [1]. Malicious Insider attacks are also becoming more widely known about, however the nature of an internal attack is not a topic that many organisations are open to discussing. This is for a variety of reasons including the risk to the company's share value and the risk of appearing to having vulnerable security, therefore making them an attractive target to other attackers. Even a company as large as a Google subsidiary: Waymo (Google's selfdrive car company) reportedly had tens of thousands of files copied from their servers and exfiltrated via a cloud storage account $[9,10]$ showing that size and resources do not offer immunity from attack.

We present here a method we are developing for the design of insider-attack detection strategies for cloud environments. In particular, our focus is on exceptionally difficult-to-detect insider attacks through forms of indirect detection. We propose a method based on attack trees for identifying strategies for combining multiple indirect measures. Specifically, the use of attack trees in this regard enables us to encapsulate all detection opportunities for insider attacks in cloud-service environments based on an attack-centric tree, which in turn more readily facilitates observations of indirect detection opportunities. Using attack trees, we hope to view an attack as a set of events in a hierarchical structure allowing the defence against an attack to be based on detecting precursor or parallel events. In this way, a culminating attack, whose individual steps were undetectable, may now become indirectly detectable.

Once we have a clear picture of the attack's properties, we propose to map them onto the Kill Chain [11]. This mapping allows us to view the attack in a chronological order, which in turn allows defence against the attack to be focused on the events surrounding any suspicious activity observed. The kill chain can then be used to examine other events later in the chain to see how far the attack has progressed and to focus resources on preventing the completion of the attack as well as determining the identity of the attacker.

The structure of the remainder of this paper is: related work on insider-attack detection and attack trees are covered in Section II. A review of our previous work on attacking virtual machines in migration as well as other attacks which are difficult to detect is 
presented in Section III. Attack trees are then outlined in Section IV and their application to virtual-machine, and other, attacks in cloud computing are discussed in Section V. Kill chains are discussed in Section VI, with their application to attack trees covered in Section VII and our conclusions in Section VIII.

\section{RELATED WORK}

Current mechanisms for detecting insiders include using Intrusion Detection Systems (which are normally focused on detecting outsiders), detecting exfiltration and monitoring for outgoing remote access [12]. These mechanisms are unsuited to detecting most insiders as they cannot allow for the knowledge that an insider possesses, which allows them to circumvent the detector in addition to exfiltrating data via alternate methods.

There are great variations in attacks that an insider can perform. In a paper on understanding and predicting the insider threat [13], Schultz asserts that there are more successful attacks coming from the inside and that there is no debate that insider-attacks pose a far greater level of risk than outsider attacks. He also asserts that, unlike external attackers, no single clue is sufficient for predicting and detecting against insiders. His paper, and a paper Bradford and $\mathrm{Hu}$ [14], discuss that intrusion detection systems (IDS) are not ideal for this role as they are primarily focused on external attackers and have a tendency to identify false positives.

The Cloud Vulnerabilities Working Group at Carnegie Mellon's CERT have released two papers on the insider-threat problem in cloud computing $[12,15]$. In the former paper, they point out the flaws in employing existing techniques for detecting in-house attackers. Using service level agreements to impose strict supply chain management, human resource requirements made contractually, transparency of security and management practices and breach notification processes are not necessarily effective at preventing or reporting insiders. The latter paper details existing media-reported attacks on cloud computing and lists insider attacks on Google Gmail, Microsoft cloud data and CyberLynk's customer data.

Attack trees are currently being used in a variety of places to assist with modelling complex systems [16, 17] and for SCADA systems [18]. In the latter paper, Ten acknowledges that attack trees are not able to offer a chronological view of a given attack. Wang et al [19] utilise attack-defence trees in order to underpin risk analysis of systems, and in particular they present an application to cloud computing. The example is a snippet of a high-level attack-defence tree with metrics for determining risk, success probability, attack cost, and impact. It is easy to see that a more in-depth version of their tree will become very complex and difficult to navigate and the metrics could easily become unwieldy.

The concept of a Kill Chain in relation to computer attacks, and to Advanced Persistent Threats (APT) in particular, was presented in a white paper by Hutchins, Clopperty and Amin [11]. The paper details APT attacks and discusses the analyses of many actual intrusions in order to determine the chain of events that lead to an attack; the purpose of this is to enable detection of an attack during its attack cycle rather than after the event. In addition, it identifies common events which indicate a potential attack is in progress, allowing detection of the attack earlier in the kill chain.

\section{III. “Undetectable” ATtACKS}

In a previous paper [20], we presented an attack on virtual machines in migration, which is extremely difficult to detect. If executed perfectly, this attack was undetectable in a laboratory environment. The attack is a wiretap on the physical copper cable that connects the server to a datastore or hypervisor. The packets are captured using a packet sniffer on a device that is unknown to the network, but attached to the network tap. Once the packets containing a virtual machine migration are captured, the packets are processed by forensic filerecovery tools. In one test, thousands of files were recovered in one migration of a Windows VM. Data from other files were recovered using the Linux string command as well as data from the clipboard.

Previous methods for detecting packet sniffers in promiscuous mode, such as poisoned ARP [21], failed to detect this attack as only one pair of the transmit and receive cables were tapped per network card. We had variable success detecting the network tap using Time Domain Reflectometry (TDR), which was able to detect the tap depending upon the length of the tap cable and its position on the host cable. Further investigations are currently being carried out to determine methods of improving the probability that cable taps formed in this way are detected.

The CERT Guide to Insider Threats [22] lists a number of case studies on insider attacks, which were extremely difficult to prevent beforehand and detect afterwards. For example, in Sabotage Case 22 , a network sniffer and a buffer overflow was used to compromise the root account allowing the malicious insider to read emails, compromise other accounts, and deface the company's website. The culprit was only revealed after they were discovered attending local organised hacking events. Theft of IP Case 5 involved outsourcing. The insider at the external company was not on their payroll and was only there to help at the request of a family member. The originating company knew nothing of their existence neutralising any personnel checks that might have taken place. Confidential material was taken and leaked, but due to the supply chain being so small, the source of the leak was located. It is not possible to eradicate this type of situation regardless of service-level agreements and due diligence. If this scenario occurred in a cloud-computing environment, detection would be significantly more complicated based on the sheer volume of insiders, the number of locations where insiders can exist and the total number of technologies involved. Based on the complex detection of these and other cases, there is no doubt that the malicious insider is getting better at hiding themselves.

These attacks, as well as our own mentioned above, are not always easy to detect directly and were only discovered after investing considerable resources costing tens of thousands of US dollars and a comprehensive digital forensic investigation. This, coupled with the jurisdictional issues presented by cloud computing, presents a very real problem in investigating attacks after the event. Whether we are able to detect our migration attack or not is less relevant than the fact that is extremely difficult to detect even when we were able to do so. Many other attacks are very difficult to detect, or detection can be unreliable and very costly. If an attack falls into these categories, they are, in all practicality, impossible to detect, hence we refer to them using the term "undetectable".

We question whether it is practical or economic to implement protection and detection strategies for every single type of attack that can be launched against the cloud-computing paradigm. Viewing the example attack at the moment of data theft, it is not possible to detect that the data is being compromised. It is unlikely that any IDS system would capture and halt this attack at that point, however, we are certain that events and actions leading up to our virtual machine attack are detectable and therefore believe it might be unnecessary to protect and defend against each attack directly. Thus, we propose an alternative to direct detection and prevention of every single attack by applying attack trees and kill chains to cloud-computing environments in order to identify indirect detection opportunities. 


\section{ATtack Trees}

An Attack Tree, as defined by Schneier [23], represents attacks as a tree structure where the root node is the goal of the attack and leaf nodes are actions or events. There can be many root nodes in a single system. If a node relies on precursor events or actions, they are expressed as sub-branches of that node, effectively making that node become an intermediate goal. It is possible to continue drilling down through the nodes ad-infinitum, however in practice, there is a natural level for an attack tree to end. This will vary depending upon the audience and the system being represented: a node called "Reboot Server" need not have further nodes for "type sudo reboot now" and "Enter Password" .

The downside to a graphical representation is that it can become difficult to express in a single view, however, this can be overcome by treating each branch as a root node. Alternatively, attack trees can also be expressed textually in a numbered set of lists. The top row of nodes having primary indexes, and each sub-node being sub-indexed and so on.

A significant benefit to using an attack tree is that critical steps are more readily identifiable. There maybe a single node which must be completed in order for a branch to have a successful outcome for the attacker. Once identified, resources can be invested in protecting the critical node instead of being spread out across a wider set of nodes.

In general terms, an attacker's preference is for a wide and flat attack tree where there are many ways of achieving each node, including the root node, as this provides the attacker with a variety of options. If one attack fails, they can try alternatives until they are successful. From a security perspective, a deep and narrow attack tree is more desirable where each node relies on several ANDed sub-nodes. This means that each node is more difficult to complete and there are more stages to complete, allowing greater detection opportunities.

\section{Applying Attack Trees to "Undetectable" Attacks}

\section{A. Defining the Attack Tree}

Using the attack on virtual machines in-transit presented in our recent work [20], we created an attack tree to examine the attack with a view to detecting precursors or key points in the attack branches which present the opportunity to predict or prevent the attack. This attack tree is part of a much larger tree we are producing, which attempts to map the attacks we are aware of for different cloud services.

Figures 1 and 2 show the attack tree for the above attack with the former being a textual view of the tree and the latter being a diagrammatic view. The fact that Node 3 is below Nodes 1 and 2 in Figure 2 is for spacial reasons; one would normally draw each level on a single plane for clarity. The italicised nodes in Figure 1 and the dotted-line nodes in Figure 2 are ones we have no direct influence over and can therefore rule them out of our consideration.

The first noticeable point in this attack tree is that there are more $A N D$ gates, which is a positive attribute from a security perspective. The attacker would prefer to have many ways (OR gates) of achieving the same goal: giving them multiple routes through the tree. In this tree, the top level nodes as well as sub-tree of Node 3 comprise almost exclusively of $A N D$ gates, meaning that any node that the provider can prevent, either at the top level or in sub-tree 3, makes the whole tree impossible.

\section{B. Traversing Trees}

Unlike a PERT (Project Evaluation and Review Technique) chart, there is no critical path through an attack tree, so it is a worthwhile
1. Initiate Migration of Desired VM or Customer Data (AND)

2. Obtain and Process Capture files (AND)

2.1. Remove Captured Files (AND)

2.1.1. Exfiltrate via Network (OR)

2.1.2. Exfiltrate via Removable Media (OR)

2.1.3. Exfiltrate via Capture Device (OR)

2.1.4. Exfiltrate via Wireless

2.2. Process Capture Files

2.2.1. Local (OR)

2.2.1.1. Obtain Forensic Apps (AND)

2.2.1.1.1. Download (OR)

2.2.1.1.2. Write Custom Apps

2.2.1.2. Introduce Forensic Apps into the System (AND)

2.2.1.3. Execute Apps

2.2.1.3.1. Opportunity to run apps (AND)

2.2.1.3.2. Rights to run apps (AND)

2.2.1.3.3. Access to PCs (AND)

2.2.1.3.4. Sufficient Storage

2.2.2. Remote

2.2.2.1. Obtain Forensic Apps (AND)

2.2.2.1.1. Download (OR)

2.2.2.1.2. Write Custom Apps

2.2.2.2. Execute Apps

2.2.2.2.1. File Recovery (OR)

2.2.2.2.2. Registry Analysis

3. Install Network Tap

3.1. Physical Access to Desired Cable(s) (AND)

3.1.1. Trial and Error (OR)

3.1.1.1. Access to Significant Proportion of Cable Infrastructure (AND)

3.1.1.2. Sufficient Storage Space (AND)

3.1.1.3. Time!

3.1.2. Understand Cable / Network Infrastructure

3.2. Tap Cable and Connect Listening Computer

3.2.1. Expose Cable and Connect Tap Device (AND)

3.2.2. Install Packet Capture Device (AND)

3.2.3. Connect Tap to Capture Device without Dropping Connection

3.3. Time!

3.4. Possession of Dedicated Hardware

3.4.1. Obtain (AND)

3.4.2. Introduce into the Organisation Covertly

Fig. 1. Textual attack tree for detecting passive network taps. Nodes in italics are beyond the control of the service provider.

exercise to examine the minimum number of nodes (stages) and the total number of paths through the tree. The organisation defending against an attack will find it more desirable to have many stages. The more stages required to perform the attack, the more work the attacker has in order to achieve the goal: the more opportunities for detection. Conversely, the greater the number of paths for the attacker, the more options they have, which is useful should one branch fail or if one is more costly (in time, money or resources) than others.

To calculate the total minimum number of stages to complete the attack, we add the minimum number of stages for each node to be completed, taking the shortest route through the tree. Node 3 has a minimum of seven stages $(3.1 .2,3.2 .1$ to $3.2 .3,3.3$ and 3.4 .1 and 3.4.2), Node 2 has a minimum of three stages (2.1.1, 2.2.2.1.1 and 2.2.2.2.1) and Node 1 is a singleton node. This gives a total minimum number of stages that the attacker needs to complete of eleven. There 


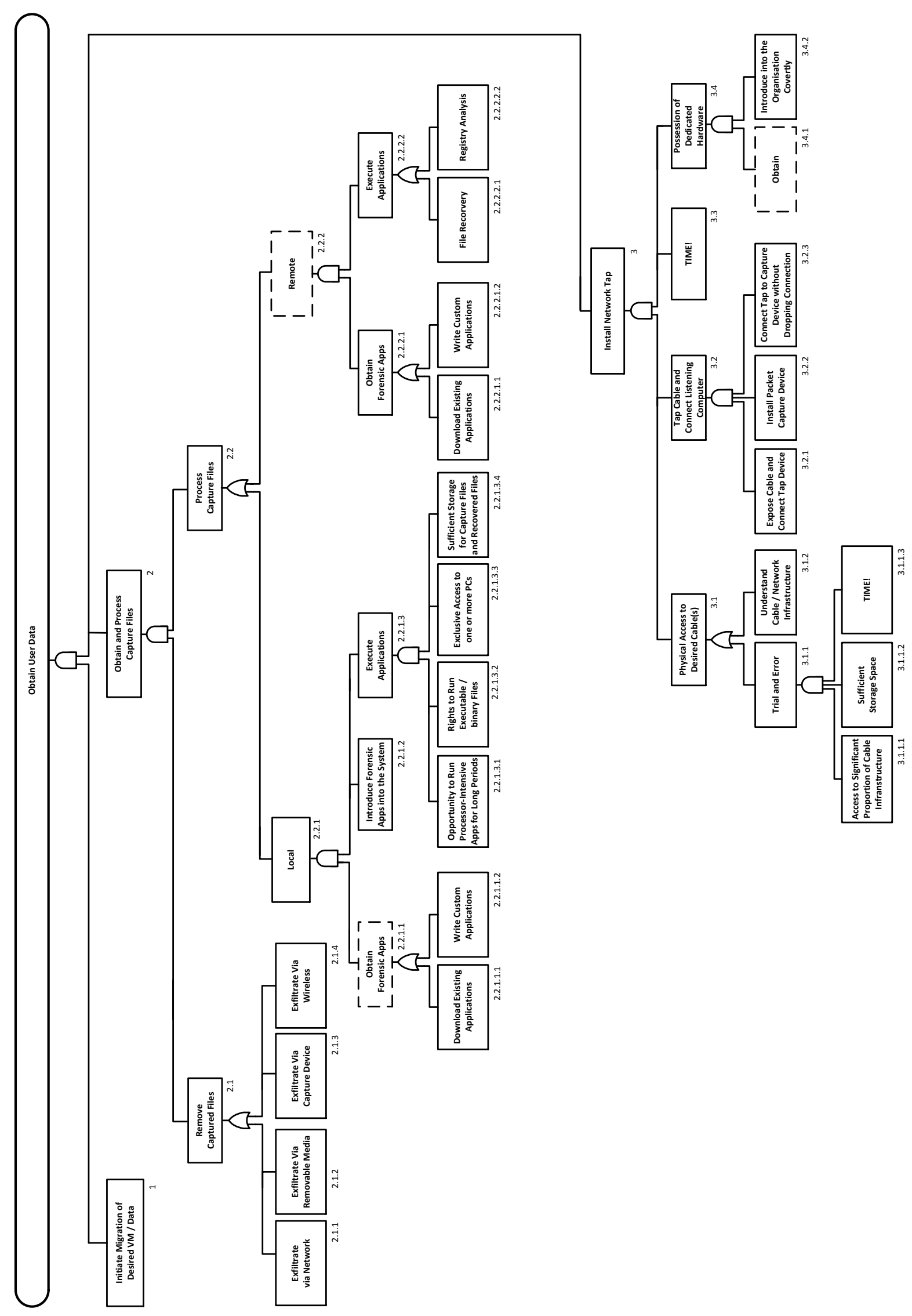

Fig. 2. Attack-Tree Diagram for Detecting Passive Network Taps 
is, however, only nine opportunities to detect or prevent this attack due to Node 2.2.2 being beyond the control of the provider.

To calculate the number of paths, the number of nodes immediately under each $O R$ gate is multiplied. Node 1 only has one path and Node 3 has only two alternative paths: either 3.1 .1 or 3.1.2. With Node 2 there are four alternatives for 2.1; Node 2.2.1 has two alternatives; 2.2.2 has two alternatives, each with two paths totalling four. The total number of paths through Node 2 is therefore four for 2.1 and eight for 2.2. By multiplying all of these together, the total number of paths can be determined: $1 \times 4 \times 8 \times 2=64$ possible paths. Despite the number of $A N D$ gates, there are still a great many possible ways of orchestrating this attack. In order to defeat the attack, we must be able to defeat or detect each path.

\section{Protection and Detection}

We use the information in Section V-B when looking to try and prevent the attack, which will ideally be in several places. By limiting the opportunity for the attack to take place in different locations of the tree, the attacker should either realise that the attack will not succeed, or they will continue with the attack. By continuing with the attack, the malicious insider will not know that their efforts are in vain, which is positive from a defensive perspective for several reasons: their efforts will not come to fruition; their continued action allows greater opportunity for detection; and these actions will yield valuable information for those defending against the attack.

The defence of the attack will vary depending upon the organisation: a government secure cloud will be unlikely to permit removable memory on-site or the introduction of hardware or software, so this attack will fail at Node 3 and reduce Node 2.1 to two opportunities from three. This also reduces the number of prevention and detection points for the defender. Viewing this as a general purpose cloud provider, it is conceivable that an insider can bring removable memory and a device, such as a Raspberry Pi, with which to perform the packet sniffing. An RJ45 terminator is sufficient to do the tapping and any sharp knife would be able to cut the outer sheath of the Cat5 e or Cat- 6 cable.

There are a variety of other key areas where we can limit the attacker's ability to perform this attack. Starting from Node 1, we address several below; listing detection and prevention opportunities.

\section{Node 1: Initiate VM migration}

Prevention: A cloud implementation where virtual machines require authorisation prior to migration will reduce this risk further.

Detection: The risk of migration attacks can be reduced by monitoring and auditing migrations. By analysing migrations to build a baseline pattern of "normal" migration behaviour, the provider will be able to look for abnormal migrations. It is possible with real-time monitoring, to detect a suspicious migration during or immediately after the migration allowing the systems administrators to determine the identity of the attacker in a timely manner.

\section{Node 2.1.1: Exfiltrate via the network}

Protection: Removal of software which enables these mechanisms will help to reduce the opportunity for malicious insiders to performs these attacks. Other methods for detecting exfiltration have been developed including SIDD [24] and MINDS [25]. There is a presumption that to exfiltrate via the network, the attacker must be able to move the data from the capture device to the network in the first instance, which may in some way be linked to the neighbouring node.

Detection: Exfiltration via the network can be achieved in a variety of ways. FTP, email, uploading files to a webserver, peer-to-peer applications, TSL / SSL connections to external computers are some of the methods that data can be exfiltrated from an organisation without the need for removable media. There are basic methods of detecting this type of exfiltration using firewalls, port blocking and centralised logging.

\section{Node 2.1.2: Exfiltrate via removable storage}

Protection: In some environments, for example, a government cloud, it is likely that the introduction of USB and other removable memory devices will be prohibited. Where USB ports and DVD drives are not explicitly required, they can be disabled (via BIOS or the OS) or physically removed from machines, further reducing the possibility of exfiltrating data this way. This node is arguably the most difficult to mitigate due to the prevalence of these devices and their integral nature in computers.

Detection: In cloud environments where the prohibition of removable media isn't mandated, the installation of removable media can be logged and monitored. USB devices can also be made read-only on specific machines allowing necessary data to be introduced, updatefiles for example, but preventing data from being taken from the machine.

\section{Node 2.1.4: Exfiltrate wirelessly}

Protection: In order to exfiltrate wirelessly, the capture device would be responsible for the transmission. As with Node 3.4.2, restricting what can be brought on-site is an effective method to protect against this mode of exfiltration.

Detection: Wireless Intrusion Prevention Systems (WIPS) can monitor the radio waves in an area looking for broadcasts that are from an unexpected source.

\section{Node 2.2.1.2: Introduce forensic applications}

Protection: The prohibiting of removable media will reduce the likelihood of forensic tools being introduced into the infrastructure, however these applications are readily available on the Internet for downloading. Established methods exist for preventing unauthorised running of binary files as shown in 2.2.1.3.

Detection: Systems administrators can perform automated searches for the file hashes of known forensic applications, which would alert them to a potential problem and allow them to investigate further.

\section{Node 2.2.1.3.1: Run processor-intensive applications}

Prevention: Once a computer is identified as being in an unacceptable state, it can be locked or the process responsible terminated. The computer can then be examined by systems administrators. Arguably, this is not direct prevention, but if this action is taken routinely when such incidents arise, insiders will know that they are unable to run processor intensive applications with malicious intentions.

Detection: Remote monitoring of computers on the network for unusual processor usage will be sufficient to detect this. Once a baselines are created for an individual computer and an individual user, detection will be obvious as this process would require running the processor at $100 \%$ for many several hours.

\section{Node 2.2.1.3.4: Obtain sufficient storage to store output}

Prevention: Preventing users from having unlimited disk space and monitoring for increases in disk usage will provide an acceptable level of protection.

Detection: Remote monitoring of computers on the network for unusual disk usage will be sufficient for detection.

\section{Node 3.1: Physical access to cables}

Prevention: Physical cabling is generally protected from external attackers, however a trusted insider is likely to be able to locate the weakest point in the network infrastructure in order to install a network tap. Restricting access to the areas containing the cabling and critical systems will reduce the opportunity for an insider to perform this attack, however, there is still the opportunity for the cabling to 
be attacked in the ceiling space, wall cavities, and similar locations. Ideally, this vulnerability would be designed out of a system before it is built. With sufficient planning and allowance for expansion, the cable system can be protected from attack by being housed in protected ducting or conduit systems and located in areas that are away from areas populated by staff and outsiders.

Detection: As detailed in our previous paper, detection of a well executed attack is difficult. Regular cable length testing using the TDR system built into many switches is one of the better methods of detection.

\section{Node 3.4.2: Introduce hardware into the system}

Prevention: As with 2.1.1.2, restricting what can be brought onto the premises is the most effective method of mitigating this attack. However, this is in itself is not a complete solution. It is to be expected that a cloud provider will have the tools required to perform the attack on-site. A Raspberry Pi and an RJ45 terminator are not unusual items at a technology company: effective administration and auditing of these objects will further reduce the likelihood of this attack being realised.

Detection: Detecting the use of these devices if present in the system is not likely to be easy. The insider will know where best to place the device and may be able to provide power in order to not have to interfere with it once planted. The most effective method of detecting this will be to focus on the exfiltration of the data that the device collects. Dust is the enemy of attacker in this case. Visual inspections of key areas looking for the disturbance of dust could lead to the detection of cable tampering.

\section{Limiting the VM Migration Attack}

Using the information in this section, we can limit the likelihood of this attack by preventing the introduction of removable media and hardware into the provider. This single control neutralises several essential Nodes - 2.1.1.2, 2.2.1.3, 3.4.2 - without which the attack will simply not be possible. It also limits the options of Node 2.1.3.

In addition, there are several key areas, which will reduce the overall likelihood of an attacker attempting this attack. By auditing and enforcing tight controls, where possible, on the initiation of migrations (Node 1), a malicious insider has reduced opportunity to migrate a VM deliberately in order to capture its data. Monitoring local disk and processor usage, the processing of captured data locally might be detected and reducing the likelihood of branch 2.1 being successful.

Controls on user storage and processor usage will allow for detection of abnormal behaviour at Nodes 2.1.4.1 and 2.1.4.2. Monitoring of binary files on computers with the organisation will reduce the opportunity for 2.1.3, which in turn reduces the whole of sub-tree 2.1. Node 3 can be addressed by prohibition of introducing hardware into the organisation, preventing 3.4.2 and protecting the cables between servers and switches will help to limit the opportunity for Node 3.1.

Using the relatively low-technology solutions outlined above, the chances of the high-technology attack being implemented are significantly reduced.

\section{Kill Chains}

Trying to build a strong defence against intrusions means that they must be examined and analysed in order to determine patterns and processes that are common to multiple attacks. This process led to a seminal White Paper by Lockheed Marting on Intrusion Kill Chains [11]. Multiple intrusions were examined and patterns were identified, which fall into seven distinct areas. Not all of these phases are completely consistent with an insider attack as they were developed with APT attacks in mind, however, the kill chain as a whole complements the attack-tree methodology already discussed. The seven phases are outlined below, with similarities and differences to their application to insiders highlighted.

Reconnaissance: Research, identification and selection of targets. With respect to insiders, this phase is any preparatory work which is required, but is not included in the following phases. This can manifest itself by insiders asking for additional access (physical or electronic), making enquiries about security mechanisms or innocuous test attacks, which may include attempting to access restricted areas in order to determine what security mechanisms are in place.

Weaponisation: For APT attacks, this is coupling a remote access Trojan with an exploit into a deliverable payload. In addition to bespoke versions, insiders could also use this as an attack vector to gain additional credentials using attack mechanisms such as Adobe Portable Document Format (PDF) or Microsoft Office documents. With respect to insider attacks, this phase is the creation of the attack device.

Delivery: In a typical network attack, the delivery method is likely to be an email attachment, malicious website, or USB removable media. These vectors are all available to a malicious insider in addition to bespoke delivery mechanisms.

Exploitation: This phase in an APT attack or an insider attack using the same attack vector is the opening of the weaponised document or email or the creation of the environment for the attack to take place. For insider attacks, this is the setting up of a suitable environment for the attack. There is an overlap with insider attacks between this phase and the Delivery and Installation phases.

Installation: For APT attacks, this is the installation of a remote access Trojan or back-door on the victim system that allows the adversary to maintain persistence inside the environment. For insider attacks, this is the actual activation of the attack. This is any action that is required to instantiate the attack after the exploitation.

Command and Control (C2): Typically for APT attacks, compromised hosts must beacon outbound to an Internet controller server to establish a C2 channel. APT malware especially requires manual interaction rather than conducting activity automatically. This will also be applicable to some insider attacks, but this phase may not be present or may be automated. With an insider attack, this phase may well be a manual interaction with hardware.

Actions on Objectives: Typically, this objective is data exfiltration which involves collecting, possibly encrypting, and extracting information from the victim environment; violations of data integrity or availability are potential objectives as well. This phase applies equally well to APT and insider attacks where the objective was to obtain data.

\section{Combining Kill Chains And Attack Trees}

Attack trees are very effective at mapping an attack, however the result does not provide a chronological perspective. This perspective would be a vital tool in analysing live attacks and determining at which point in the process the attack was discovered. We propose combining attack trees with kill chains to provide a complete and chronological picture of an attack as shown in Figure 3.

By allocating each step of the attack to its relevant category within the kill chain, the attack tree gains a chronological order from left to right. Within each phase of the kill chain, chronology remains left to right as with PERT charts, however, where there is any ambiguity between logic associations and chronology, nodes can be situated vertically (as with Node 3.2 in Figure 3), alternatively, directional arrows can be added between nodes to show dependant tasks. 


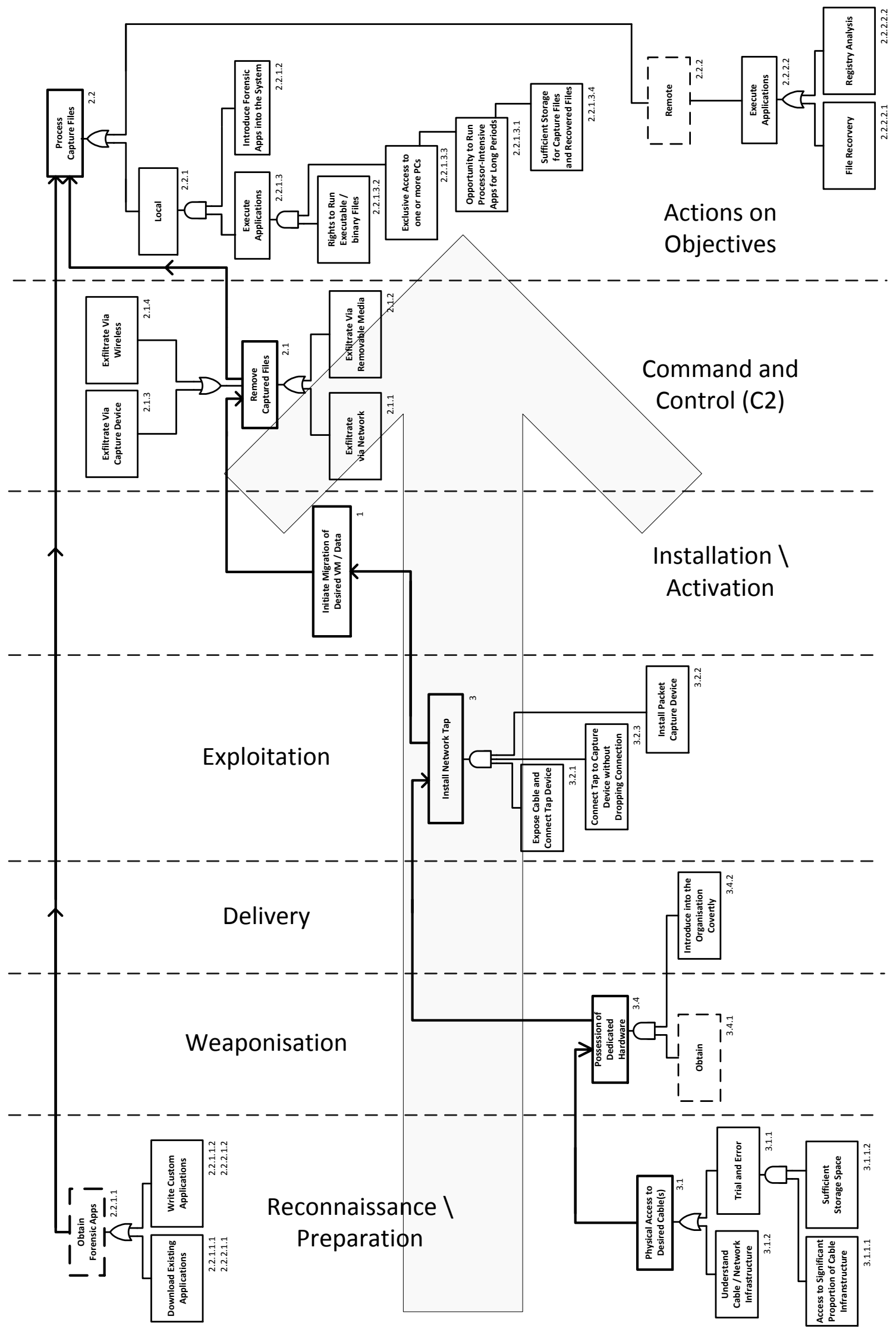

Fig. 3. Attack Tree Kill Chain Diagram for Detecting Passive Network Taps 
The picture that the Attack Tree Kill Chain provides is multipurpose and bi-directional. If an attack or suspicious activity is detected, it can be plotted onto the attack tree in the kill chain to see exactly how far into the attack the detection has occurred. The organisation can then begin to examine events later in the kill chain allowing them to determine whether data loss has occurred and, if not, allowing them to halt the attack using the predefined protection and detection strategies. The Attack Tree Kill Chain can also be examined in reverse to see what actions can be taken in future to defend against a repeat attack, allowing the organisation to move detection further up the kill chain and arrest attacks in a more timely manner.

Another benefit to the Attack Tree Kill Chain is that critical nodes become more clear. In Figure 3, there is a single node in the Installation / Activation phase. This node is therefore critical to the whole attack. Identifying this critical node allows for concentrated detection and protection.

\section{CONCLUSION}

We have demonstrated here that, although a single attack event may be very difficult or impossible to detect, by examining the circumstances around that attack, we can look for precursors and dependencies. By identifying these other events, we can detect attacks vicariously and effectively. In order to identify these precursors and dependencies, we used attack trees to map all of the known events and activities surrounding an attack. The attack tree allowed us to examine the system at a higher level and look possible ways of achieving the high level goal, then drill down further into the attack looking for those dependencies and precursors with a view to detecting those that we cannot protect against.

Examining the attack presented at TrustCom 2013 using attack trees, we were able to identify several key nodes where this attack can be prevented or detected. This can vary depending on the cloud deployment model, but even in a less secure environment, there are several mitigation opportunities, which if targeted effectively, can make the attack increasingly difficult to achieve. Where a limited budget exists, the use of attack trees in this way can help to focus energy and money where it can have the greatest impact.

Mapping the events from the attack tree to the kill chain, we are able to put the attack into chronological order by placing each node into the appropriate kill-chain phase. When suspicious activity is detected, the kill chain can be referenced to see where in the chain that activity is located. The chain can then be followed to see if there is evidence of supporting activity as well as activity later in the chain. Where appropriate, actions can be taken to intercept an attack at a given point or to pursue further evidence with a view to attribution. The combined use of attack trees with the kill chain allows for a much more complete perspective of an attack. This perspective improves the general understanding of the attack as well as helping to clearly identify critical nodes. This understanding in-turn simplifies detection and prevention opportunities.

\section{REFERENCES}

[1] R. C. Team, "Lessons from the Cryptojacking Attack at Tesla," Online, 20, February 2018, https://blog.redlock.io/ cryptojacking-tesla Last Accessed 23 February, 2018.

[2] P. Institute, "2011 Cost of Data Breach Study: United States," Online, March 2012, www.ponemon.org/local/upload/file/2011_ US_CODB_FINAL_5.pdf Last Accessed 05 June, 2013.
[3] V. Enterprise, "2017 Data Breach Investigations Report,” Online, 2017, http://www.verizonenterprise.com/verizon-insightslab/dbir/2017/ Last Accessed 05 June, 2017.

[4] — , "2013 Data Breach Investigations Report," Online, 2013, http://www.verizonenterprise.com/resources/reports/ rp_data-breach-investigations-report-2013_en_xg.pdf Last Accessed 05 June, 2017.

[5] P. Institute, "2012 Cost of Cyber Crime," Online, 7 November 2012, http://www.tik.ee.ethz.ch/education/lectures/ManSec/ Exercises/us-ponemon-survey-results-2012-hp-v1_9123.pdf Last Accessed 5 June, 2017.

[6] _ _ "2016 Cost of Cyber Crime Study," Online, October 2019, http://www.ponemon.org/local/upload/file/2016\%20HPE\% 20CCC\%20GLOBAL\%20REPORT\%20FINAL\%203.pdf Last Accessed 5 June, 2017.

[7] R. Richardson, "2010 / 2011 CSI Computer Crime and Security Survey," Online, 5 April 2011, http://gocsi.com/survey Last Accessed 04 June, 2013.

[8] Ponemon Institute , "2016 Cost of Cyber Crime Study," Online, 5 October 2017, https://www.accenture.com/us-en/insight-costof-cybercrime-2017 Last Accessed 15 April, 2018.

[9] Reuters, "Before lawsuit, uber fell out with 'big brother' Google, Kalanick testifies," Online, 7, February 2018, https://www.reuters.com/article/us-alphabet-uber-trial/beforelawsuit-uber-fell-out-with-big-brother-google-kalanicktestifies-idUSKBN1FR1ON Last Accessed 13 April, 2018.

[10] S. Friedberg, "Sumary Report - Project Unicorn Investigation," Online, 5, August 2016, https://Fwww.courthousenews.com/wpcontent/uploads/2017/10/Waymo-Uber-Due-DiligenceSummary.pdf Last Accessed 13 April, 2018.

[11] E. Hutchins, M. Clopperty, and R. Amin, "Intelligence-Driven Computer Network Defense Informed by Analysis of Adversary Campaigns and Intrusion Kill Chains," in Proceedings of the 6th International Conference on Information Warfare and Security, Academic Conferences Limited, March, 2011, pp. 113-125.

[12] D. Cappelli, R. Trzeciak, and R. Floodeen, "The Key to Successful Monitoring for Detection of Insider Attacks," Online, 5 April 2010, www.cert.org/archive/pdf/Insider-Threat-RSA2010.pdf Last Accessed 18 June, 2013.

[13] E. Schultz, "A Framework for Understanding and Predicting Insider Attacks," Computers \& Security, vol. 21, no. 6, pp. 526531, 2002.

[14] P. Bradford and N. Hu, "A Layered Approach to Insider Threat Detection and Proactive Forensics," in Proceedings of the Twenty-First Annual Computer Security Applications Conference (Technology Blitz), 2005.

[15] R. Ko and S. Lee, "Cloud Computing Vulnerability Incidents: A Statistical Overview," 23 August 2012, https://cloudsecurityalliance.org/download/cloudcomputing-vulnerability-incidents-a-statistical-overview Last Accessed 18 June, 2013.

[16] C. Fung and others., "Survivability Analysis of Distributed Systems Using Attack Tree Methodology," in Military Communications Conference, 2005. MILCOM 2005. IEEE, vol. 1, 2005, pp. 583-589.

[17] R. Dewri and others., "Optimal Security Hardening on Attack Tree Models of Networks: a Cost-Benefit Analysis," International Journal of Information Security, vol. 11, no. 3, pp. 167-188, 2012. [Online]. Available: http://dx.doi.org/10. 1007/s10207-012-0160-y

[18] C. Ten, C. Liu, and M. Govindarasu, "Vulnerability Assessment 
of Cybersecurity for SCADA Systems Using Attack Trees," in Power Engineering Society General Meeting, 2007. IEEE. IEEE, 2007, pp. 1-8.

[19] P. Wang and others., "Threat Risk Analysis for Cloud Security Based on Attack-Defense Trees," in Computing Technology and Information Management (ICCM), 2012 8th International Conference on, vol. 1. IEEE, 2012, pp. 106-111.

[20] A. Duncan, S. Creese, and M. Goldsmith, "Cloud Computing: Insider Attacks on Virtual Machines During Migration," in Trust, Security and Privacy in Computing and Communications (TrustCom), IEEE 11th International Conference on, 2013.

[21] D. Sanai, "Detection of Promiscuous Nodes Using ARP Packets," A White Paper from http://www.securityfriday.com, 2002, http://securityfriday.com/promiscuous_detection_01.pdf Last Accessed 25 February, 2013.

[22] D. Cappelli, A. Moore, and R. Trzeciak, The CERT Guide to Insider Threats: How to Prevent, Detect, and Respond to Information Technology Crimes (Theft, Sabotage, Fraud). AddisonWesley Professional, 2012.

[23] B. Schneier, “Attack Trees,” Dr. Dobb's Journal, vol. 24, no. 12, pp. 21-29, 1999.

[24] L. Yali, "SIDD: A Framework for Detecting Sensitive Data Exfiltration by an Insider Attack," Hawaii International Conference on System Sciences, vol. 0, pp. 1-10, 2009.

[25] E. Eilertson, L. Ertoz, V. Kumar, and K. Long, "MINDS: A New Approach to the Information Security Process," DTIC Document, Tech. Rep., 2004. 